\title{
A new Stark decelerator based surface scattering instrument for studying energy transfer at the gas-surface interface
}

Cite as: Rev. Sci. Instrum. 86, 043306 (2015); https://doi.org/10.1063/1.4918797

Submitted: 18 February 2015 . Accepted: 12 April 2015 . Published Online: 24 April 2015

Daniel P. Engelhart, Fabian Grätz, Roman J. V. Wagner, Henrik Haak, Gerard Meijer, Alec M. Wodtke, and Tim Schäfer

\section{ARTICLES YOU MAY BE INTERESTED IN}

Velocity Distributions in Molecular Beams from Nozzle Sources

The Physics of Fluids 8, 780 (1965); https://doi.org/10.1063/1.1761320

Ion and velocity map imaging for surface dynamics and kinetics

The Journal of Chemical Physics 147, 013939 (2017); https://doi.org/10.1063/1.4983307

Velocity map imaging of ions and electrons using electrostatic lenses: Application in photoelectron and photofragment ion imaging of molecular oxygen

Review of Scientific Instruments 68, 3477 (1997); https://doi.org/10.1063/1.1148310

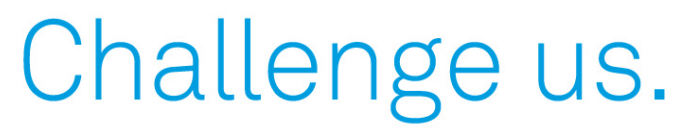

What are your needs for periodic signal detection?

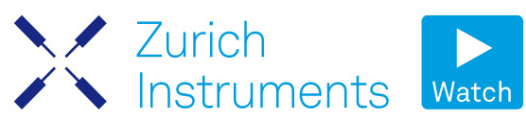

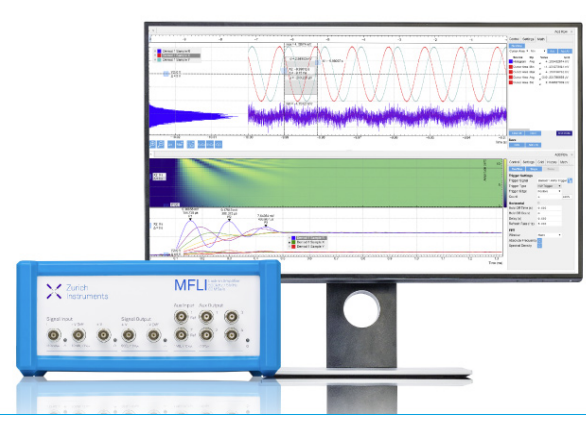

86, 043306 


\title{
A new Stark decelerator based surface scattering instrument for studying energy transfer at the gas-surface interface
}

\author{
Daniel P. Engelhart, ${ }^{1,2, a)}$ Fabian Grätz, ${ }^{1,2, a)}$ Roman J. V. Wagner, ${ }^{1,2}$ Henrik Haak, ${ }^{3}$ \\ Gerard Meijer, ${ }^{4}$ Alec M. Wodtke, ${ }^{1,2}$ and Tim Schäfer ${ }^{1,2, b)}$ \\ ${ }^{1}$ Institute for Physical Chemistry, Georg-August University of Göttingen, Tammannstraße 6, \\ 37077 Göttingen, Germany \\ ${ }^{2}$ Department of Dynamics at Surfaces, Max Planck Institute for Biophysical Chemistry, Am Faßberg 11, \\ 37077 Göttingen, Germany \\ ${ }^{3}$ Fritz Haber Insitute of the Max Planck Society, Faradayweg 4-6, 14195 Berlin, Germany \\ ${ }^{4}$ Radboud University, 6500 HC Nijmegen, The Netherlands
}

(Received 18 February 2015; accepted 12 April 2015; published online 24 April 2015)

\begin{abstract}
We report on the design and characterization of a new apparatus for performing quantum-state resolved surface scattering experiments. The apparatus combines optical state-specific molecule preparation with a compact hexapole and a Stark decelerator to prepare carrier gas-free pulses of quantum-state pure $\mathrm{CO}$ molecules with velocities controllable between 33 and $1000 \mathrm{~m} / \mathrm{s}$ with extremely narrow velocity distributions. The ultrahigh vacuum surface scattering chamber includes homebuilt ion and electron detectors, a closed-cycle helium cooled single crystal sample mount capable of tuning surface temperature between 19 and $1337 \mathrm{~K}$, a Kelvin probe for non-destructive work function measurements, a precision leak valve manifold for targeted adsorbate deposition, an inexpensive quadrupole mass spectrometer modified to perform high resolution temperature programmed desorption experiments and facilities to clean and characterize the surface. () 2015 AIP Publishing LLC. [http://dx.doi.org/10.1063/1.4918797]
\end{abstract}

\section{INTRODUCTION}

Molecule-surface scattering experiments are a useful tool for investigating dynamics at the gas-surface interface. In particular, energy transfer processes occurring during molecular collisions with the surface can be studied quantum-state selectively when combining surface scattering experiments with high resolution spectroscopy. ${ }^{1-4}$ In these experiments, the quantum state of the molecule is determined before and after the scattering event, allowing a direct assignment of energy transfer pathways. Using optical methods, the impinging molecule can be prepared in excited states, providing the possibility to study the influence of electronic, vibrational, and rotational excitation on energy transfer at surfaces. In particular, vibrational energy transfer at surfaces is crucial for bond-breaking and -making processes in surface reactions, which has triggered a variety of experimental and theoretical studies. ${ }^{5-12}$

In contrast, surface scattering of electronically excited molecules has been less extensively investigated, mostly due to their challenging preparation in the laboratory. Recently, we constructed a new surface scattering apparatus with which we scattered $\mathrm{CO}$ molecules prepared in the metastable $\mathrm{a}^{3} \Pi$ state (hereafter referred to as $\mathrm{CO}^{*}$ ) from a $\mathrm{Au}(111)$ surface and monitored resultant electron emission. We observe a strong influence of molecular vibration and surface temperature on the electron emission yield, which we describe using an anion mediated de-excitation mechanism. ${ }^{13,14}$

\footnotetext{
a) D. P. Engelhart and F. Grätz contributed equally to this work.

b) Author to whom correspondence should be addressed. Electronic mail: tschaef4@gwdg.de
}

In this article, we describe in detail the design, construction, and performance of this new apparatus for studying energy transfer at the solid-gas interface with precise control of incident molecule's electronic, vibrational, rotational, and translational energy, as well as surface temperature, orientation, and composition. First, we give an overview of the molecular beam source including beam source, compact hexapole filter, and 131 stage Stark decelerator. Then, we offer an overview of the surface scattering chamber, including construction and performance details of the sample mount, charged particle detector, Kelvin probe, and temperature programmed desorption (TPD) setup.

In order to demonstrate the versatility and performance of this machine, we report the acceleration and deceleration of $\mathrm{CO}^{*}$ molecules between 33 and $512 \mathrm{~m} / \mathrm{s}$ and detection of electrons emitted when the electronically excited molecules quench on the $\mathrm{Au}(111)$ surface. Additionally, we demonstrate the great variety of surface preparation and characterization that is possible using this machine. We report the evolution of the work function of the $\mathrm{Au}(111)$ surface versus adsorbate coverage for adsorbed noble gases $\mathrm{Ar}, \mathrm{Kr}$, and $\mathrm{Xe}$. Exact coverage information and adsorbate binding energy are extracted via TPD.

\section{EXPERIMENTAL SETUP}

\section{A. Overview}

Figure 1 shows a cutaway three dimensional model of the molecular beam surface scattering apparatus. The entire apparatus consists of three differentially pumped vacuum chambers connected to a UHV surface scattering chamber. 


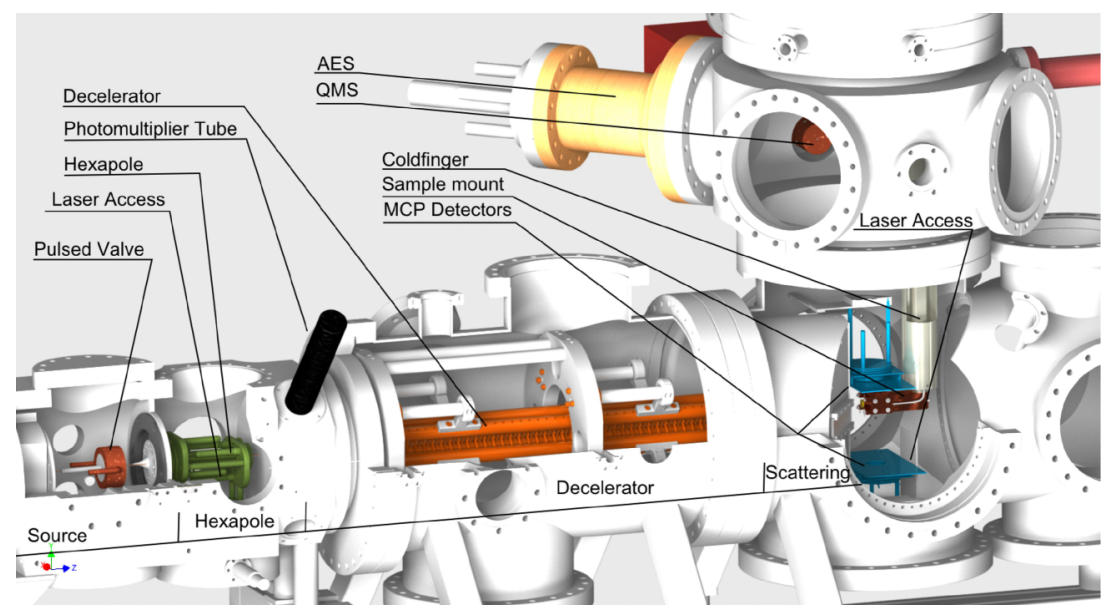

FIG. 1. Cutaway drawing of surface scattering machine. Four differentially pumped chambers: the source, hexapole, decelerator, and scattering chamber comprise the instrument. A beam of $\mathrm{CO}$ is formed with a pulsed valve. After passing a skimmer, $\mathrm{CO}$ is excited with a laser light pulse to the $\mathrm{a}^{3} \Pi\left(\mathrm{CO}^{*}\right)$ state and deflected by $3.5^{\circ}$ with an off-axis hexapole. The phosphorescence of $\mathrm{CO}^{*}$ emerging from the hexapole is monitored with a photomultiplier tube. The $\mathrm{CO}^{*}$ 's velocity is then manipulated with the decelerator before it enters the scattering chamber to collide with a prepared surface. Microchannel plate (MCP) detectors for ions and electrons are also shown. Surface composition is examined with an Auger electron spectrometer (AES). A quadrupole mass spectrometer (QMS) is used to perform temperature programmed desorption.

On the far left of the drawing is the molecular beam source chamber, where a cooled pulsed valve (General Valve Series 99 ) is mounted. The source chamber is connected via a sharp edged conical skimmer (Beam Dynamics, model 2, Ni, $2 \mathrm{~mm}$ aperture) to the hexapole chamber, second from left. The hexapole chamber is separated from the third differential pumping chamber, which houses the molecular decelerator, by a $2 \mathrm{~mm}$ thin aperture. The decelerator chamber is connected to the UHV chamber by a $2 \mathrm{~mm}$ aperture. Every component in the apparatus has been designed to survive a bake out of 24-48 $\mathrm{h}$ at $100^{\circ} \mathrm{C}$. The source and decelerator chamber are evacuated via Pfeiffer Vacuum HiPace 700 turbomolecular pumps (TMPs) with a pumping speed of $685 \mathrm{~L} / \mathrm{s}$. The hexapole chamber is evacuated by a smaller HiPace $400 \mathrm{TMP}$ at $355 \mathrm{~L} / \mathrm{s}$. The scattering chamber is pumped by a HiPace 700 TMP backed by a smaller HiPace 80 TMP with a pumping speed of $67 \mathrm{~L} / \mathrm{s}$ in series after the larger pump. All TMPs are backed by 3 Pfeiffer MVP-070-3 diaphragm pumps $\left(4.3 \mathrm{~m}^{3} / \mathrm{h}\right)$ in parallel, maintaining a forevacuum pressure of $10^{-1}$ Torr. Pumping speeds are given for $\mathrm{N}_{2}$. The scattering chamber is maintained at a pressure of less than $10^{-10}$ Torr with no detectable pressure rise when the molecular beam is on.

\section{B. Production of state selected velocity controlled carrier gas-free molecular beams}

\section{Supersonic molecular beam and hexapole state selection}

We produce a pulse of $\mathrm{CO}$ molecules with a narrow distribution of vibrational and rotational states by expanding a mixture of $20 \% \mathrm{CO}$ in Xe or Ar into vacuum at 1 bar stagnation pressure through the $0.76 \mathrm{~mm}$ diameter orifice of a pulsed valve at $10 \mathrm{~Hz}$. The pulsed valve has been modified so that the tension of the spring controlling the force of the poppet pushing against the orifice can be adjusted via a micrometer outside of the vacuum chamber, making the valve suitable for use over a wide range of temperatures. Valve temperature is controlled by flowing nitrogen gas through a copper coil submerged in liquid nitrogen and subsequently through an inline resistance heater. The nozzle temperature is stabilized by referencing a proportional integral differential (PID) controller (Eurotherm 2408) to a K-type thermocouple on the face of the valve. This PID controller controls the heating current sent to the inline heater to produce a constant flow of cool nitrogen. In this way, the temperature of the valve can be controlled within $\pm 0.5 \mathrm{~K}$ for 10-12 h using $10 \mathrm{~L}$ of liquid $\mathrm{N}_{2}$. Cooling the nozzle to $260 \mathrm{~K}$ or $101 \mathrm{~K}$ for $\mathrm{CO}$ in $\mathrm{Xe}$ or Ar, respectively, produces a gas pulse with a most probable velocity of $360 \mathrm{~m} / \mathrm{s}$.

The entire nozzle assembly is mounted on one of two specially built flanges which can be reproducibly positioned on the source chamber using two alignment pins. One flange mounts the pulsed valve at a $3.5^{\circ}$ angle relative to the main beam axis. Mounting the valve in this orientation is hereafter referred to as the bent geometry. The second flange mounts the valve on the axis of the decelerator (linear geometry). In addition to the two flanges, the wall between the first and second differential pumping regions of the machine is removable and features a mount for a skimmer. Upon changing the geometry of the pulsed valve, one also swaps out the differential pumping wall such that the skimmer is also in a bent or linear geometry. Producing the molecular beam in the bent geometry enables production of a quantum-state pure, carrier gas-free molecular beam of $\mathrm{CO}^{*}$ as described in detail below. The apparatus is most often operated with the nozzle in the bent geometry.

Immediately after passing through the skimmer, the molecular beam is crossed with a $206 \mathrm{~nm}$ laser pulse with approximately $10 \mathrm{~ns}$ duration, a pulse energy of about $2 \mathrm{~mJ}$, and a bandwidth of $300 \mathrm{MHz}$ before entering a compact hexapole. The laser crosses the molecular beam via $16 \mathrm{~mm}$ UHV fused silica laser windows mounted at Brewster's angle. The laser pulse is generated by mixing the 4th harmonic of a Nd:YAG (Spectra Physics, Quanta-Ray Lab 170-10) pulsed laser with the output of a narrow bandwidth all solidstate injection seeded optical parametric oscillator which is pumped with the 2nd harmonic of the same Nd:YAG laser 


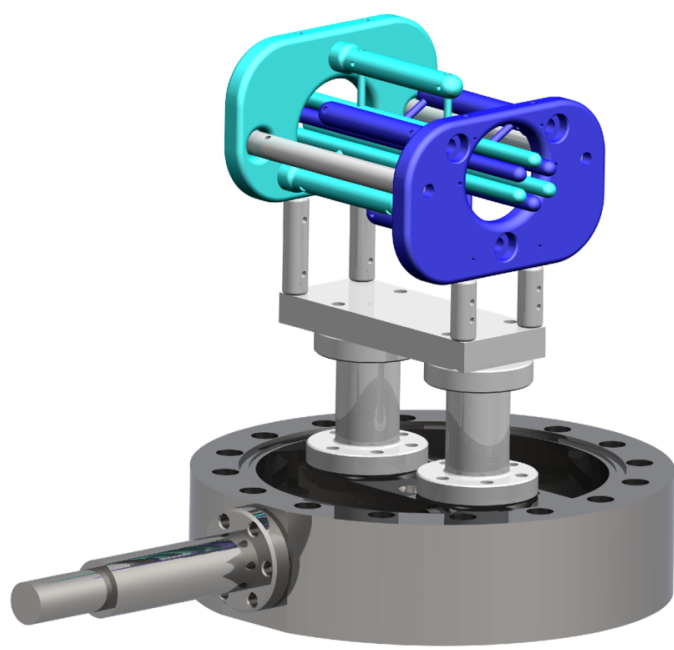

FIG. 2. CAD model of hexapole mounted on CF 100 flange. In the drawing, MACOR pieces are white and the two stainless steel 3-rod pieces are shaded blue. The entire construction is mounted on a micrometer driven translation stage to optimize transverse position during operation. This design allows exceptionally high electric fields to be used without discharging. Hence, the hexapole can be only $110 \mathrm{~mm}$ long.

in a BBO crystal. The laser system has been described elsewhere. ${ }^{15} \mathrm{CO}^{*}$ is prepared via the $\mathrm{a}^{3} \Pi_{1}(v=0, J=1)$ $\leftarrow \mathrm{X}^{1} \Sigma^{+}(v=0, J=1)$ transition at $206 \mathrm{~nm}$. CO* has a $2.6 \mathrm{~ms}$ lifetime, a dipole moment of $1.37 \mathrm{D}$, and exhibits a linear Stark effect, all properties suitable for manipulation with electric fields. When $\mathrm{CO}^{*}$ in a low field-seeking quantum state enters an electric field it will gain Stark energy. It will then be focused toward a field minimum in order to minimize internal energy. This is the general principle with which an electrostatic hexapole focuses neutral polar molecules.

After laser excitation, the hexapole voltage is switched on at $\pm 14 \mathrm{kV}$ and the electronically excited molecules are bent by $3.5^{\circ}$ and pass through a $2 \mathrm{~mm}$ aperture into the third differential pumping chamber containing the Stark decelerator. Shown in Figure 2 is a computer aided design (CAD) drawing of the hexapole. The hexapole utilizes a unique design by mounting each of the three rods of like polarity onto a highly polished stainless steel plate. The two 3-rod pieces are then mounted on individual MACOR ceramic supports opposite one another. The two 3-rod stainless steel pieces are shown in shades of blue while all ceramic parts are shown in white. The entire construction is mounted on a micrometer driven translation stage for adjustment of the transverse position of the hexapole for optimal coupling into the Stark decelerator.

This design has several advantages. Maximum operating voltage is limited by surface discharge via the insulator in which the electrodes are mounted. Using our design, the minimum surface distance between electrodes is $75 \mathrm{~mm}$, allowing operation at $\pm 14 \mathrm{kV}$. At this voltage, the hexapole need only be $110 \mathrm{~mm}$ long to deflect a beam of CO* with a mean velocity of $360 \mathrm{~m} / \mathrm{s}$ the required $3.5^{\circ}$ through the $2 \mathrm{~mm}$ differential pumping aperture.

Figure 3 (left panel) shows simulated trajectories of $\mathrm{CO}^{*}$ molecules, red dots, and carrier gas, blue dots, entering the hexapole at $3.5^{\circ}$ relative to the decelerator axis. The simulation shown is in the plane of tilt. It clearly shows that while nearly all of the electronically excited molecules are guided into the next chamber, only a small fraction of the carrier gas in the beam pulse is transmitted through the hexapole chamber. Trajectory simulations perpendicular to the plane of tilt (not shown) also indicate near perfect transmission of CO* through the hexapole chamber. The right hand panel of Figure 3 is an experimental measurement of the intensity of the molecular beam pulse after the hexapole chamber as a function of the hexapole voltage. These measurements were made after optimizing hexapole on- and off-switching times by recording signal from the impinging metastable beam on a microchannel plate (MCP) detector mounted directly downstream from the hexapole chamber. Signal intensity is enhanced by a factor of 21 when the hexapole is switched at $\pm 14 \mathrm{kV}$. The hexapole acts as a filter for those excited molecules in a low field-seeking state.

When nozzle and skimmer are in the linear configuration, the $\mathrm{CO}$ beam can be seeded in a light carrier gas such as $\mathrm{H}_{2}$ or He to produce much faster beams. Although the carrier gas cannot be removed from the beam, at mean initial velocities of up to $1000 \mathrm{~m} / \mathrm{s}$ the electronically excited molecules can be accelerated or decelerated with respect to the carrier gas, thereby separating them in time and space.

\section{Acceleration and deceleration}

After being focused into the third differentially pumped vacuum chamber, the molecular beam pulse enters the Stark decelerator. At the entrance to the decelerator chamber, we
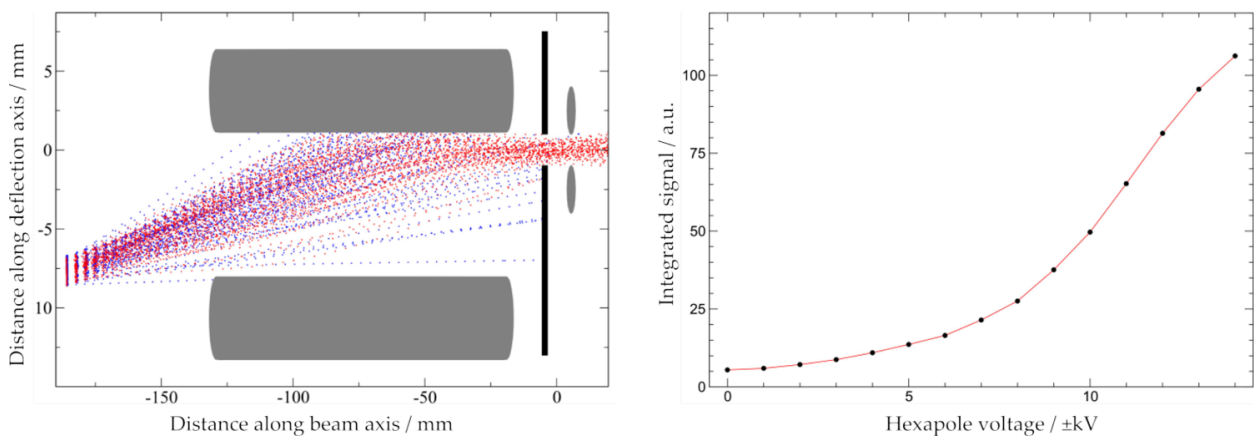

FIG. 3. Left panel: trajectory simulation of $\mathrm{CO}^{*}$ (red) and carrier gas (blue) traveling through hexapole with pulsed valve mounted at $3.5^{\circ}$ relative to beam axis. Right panel: measured transmission of $\mathrm{CO}^{*}$ through second differential pumping chamber as function of hexapole voltage. 


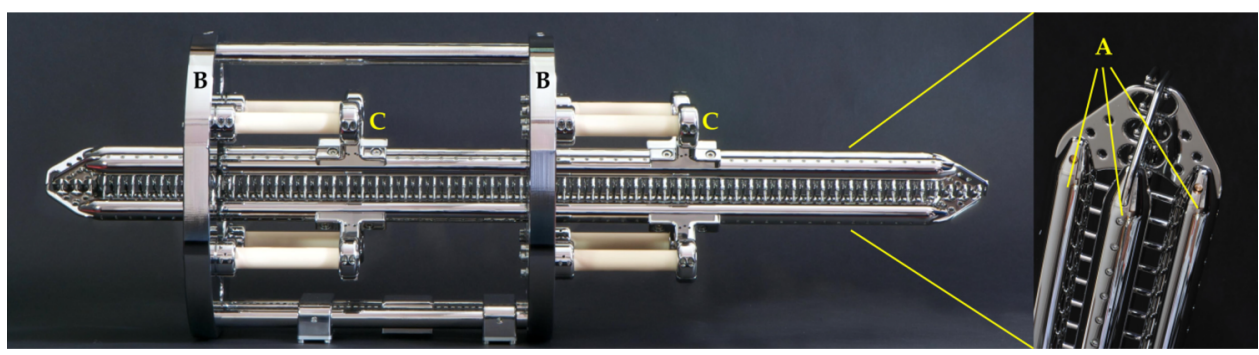

FIG. 4. The left panel shows the fully bakeable Stark decelerator. Each of four stainless steel mounting rods (A) is affixed to two mounting plates (B) via two MACOR rods (C) mounted in the same orientation, allowing the device to be baked at $100^{\circ} \mathrm{C}$ without inducing undue mechanical stress. The right panel shows a close-up of the perpendicular electrode pairs constructed by mounting four stainless steel mounting rods and specially designed end pieces which allow for a very short distance from the last decelerator electrode pair to the surface scattering chamber. Photographs by Georg Heyne, FHI Berlin.

monitor the phosphorescence of the $\mathrm{CO}^{*}$ molecules via a solar blind photomultiplier tube (PMT, Hamamatsu R821, $160 \mathrm{~nm}-320 \mathrm{~nm}$, gain: $4 \times 10^{5}$ ).

Stark deceleration has been previously reviewed in detail in several publications. ${ }^{16-19}$ The following is a brief description of the construction and operation of our Stark decelerator-see Figures 4 and 5. The decelerator is made up of 131 deceleration stages comprised of 132 equidistant pairs of parallel $3 \mathrm{~mm}$ diameter cylindrical electrodes with hemispherical caps. The distance between paired electrodes is $5 \mathrm{~mm}$, center to center. Each pair is spaced $L=5.5 \mathrm{~mm}$ apart, center to center, and positioned perpendicular to the pair on either side. Each electrode is very carefully polished to allow for fast switching between either $16.5 \mathrm{kV}$ or $-16.5 \mathrm{kV}$ and ground with no electric discharge. All electrodes with the same orientation are switched simultaneously. That is to say, while all vertical electrodes are grounded, each horizontal pair is at high voltage and vice versa. As shown in Figure 4 , all electrodes pointing in the same direction are connected to a single polished stainless steel rod. In order to place an electrode pair at high voltage under operating conditions, one places the two rods opposite each other at high voltage, one at $16.5 \mathrm{kV}$, the other at $-16.5 \mathrm{kV}$. The remaining two rods are kept at ground potential.

The Stark decelerator takes advantage of the Stark effect on $\mathrm{CO}^{*}$ in inhomogeneous time-varying electric fields. As a CO* molecule in a low field-seeking state travels from the hexapole chamber into the decelerator, all electrodes are at ground potential. When the molecule is between the first and second electrode pair, the electrode pair immediately downstream from the molecule is switched to high voltage, as shown in the left panel of Figure 5. As the molecule travels toward the high voltage electrodes, the magnitude of the field surrounding the molecule increases and the molecule's Stark energy, $W(z)$, increases. The molecule gains potential energy. This increase in the molecule's Stark energy is balanced by a decrease in translational energy; the molecule is slowed. At some point before the beam pulse reaches the midpoint between the two charged electrodes, on the rising slope of the potential hill, the electrodes are returned to ground and the next electrode pair is placed at high voltage, see Figure 5, right panel. The molecule then experiences another potential energy hill and so on. In this way, by switching the electrodes such that $W(z)$ is shifted synchronously with the movement of the pulse of molecules through the decelerator, each molecule can lose up to $1 \mathrm{~cm}^{-1}$ of energy per electrode stage. ${ }^{17}$

The magnitude of translational energy that the molecule loses is dictated by its position relative to the electrodes when the fields are switched. Using terms developed in accelerator physics, the phase angle is defined as $\varphi=2 \pi\left(\frac{z}{2 L}\right)$ where $z$ is the molecule's position along the beam axis in units of $L$. Switching the fields when the molecule is at the position of maximum electric field corresponds to a phase angle of $\varphi=90^{\circ}$.

By timing the switches such that the electrodes are placed at high voltage after the beam pulse has flown past, the molecular beam can also be accelerated in an analogous fashion. Such timing schemes are referred to with negative phase angles. As the molecules fly toward each charged electrode pair, the field minimum is at the midpoint between the two electrodes. Therefore, there is a force driving the
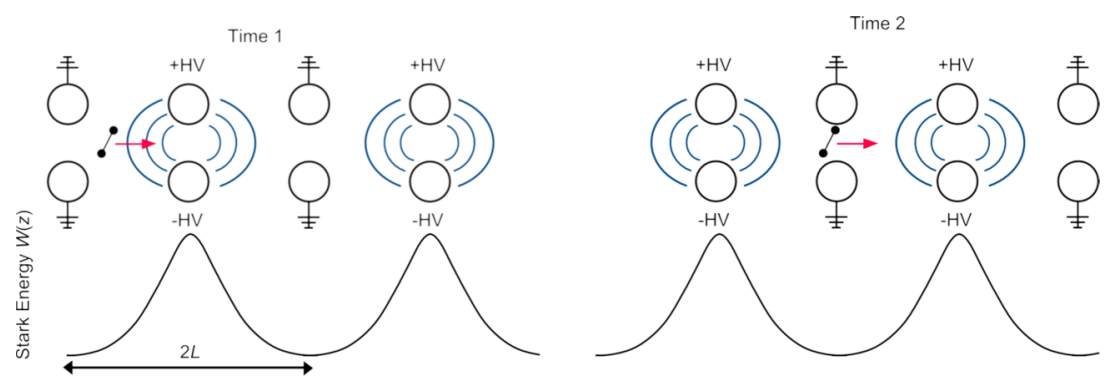

FIG. 5. Deceleration scheme of a Stark decelerator. Stark energy plotted against longitudinal position of CO* molecule in the decelerator. The Stark energy has a period of $2 L$. Molecules fly towards charged electrodes, (Time 1) increasing Stark energy and losing translational energy. At some point before molecules pass between charged electrode pair, electrodes are switched from high voltage to ground, and vice versa, (Time 2) and the process begins again. 
low field-seeking molecules toward the molecular beam axis and the molecules are spatially focused in one or the other transverse direction at each deceleration stage. This transverse focusing is beneficial as the distance from pulsed valve to surface is $96 \mathrm{~cm}$.

The decelerator can also be operated in guiding mode, $\varphi=0^{\circ}$, in which the velocity is not changed. Rather, the pulse is guided through the chamber at the initial velocity of $360 \mathrm{~m} / \mathrm{s}$. For this mode of operation, the decelerator is switched in so-called $s=3$ mode. Switching the voltages as the molecular beam pulse passes between each electrode stage is referred to as $s=1$ mode. Switching in $s=3$ mode simply switches electrodes at $1 / 3$ the frequency of $s=1$. Operating the decelerator in $s=3$ mode allows the beam pulse to travel between one energized electrode pair between each switch. The resultant increased transverse focusing results in a denser beam pulse leaving the decelerator and enhances signal significantly. Because it effectively results in only $1 / 3$ of the possible deceleration stages, we use $s=3$ mode only for guiding.

In order to achieve UHV conditions necessary for surface scattering experiments, we bake the surface and decelerator chambers to around $100{ }^{\circ} \mathrm{C}$ for $24-48 \mathrm{~h}$. This was not possible in any previously reported decelerator designs. As shown in Figure 4, each stainless steel rod to which the electrodes are attached is connected via two ceramic rods to a polished stainless steel support frame. Our design mounts the decelerator using ceramic rods that all point in the same direction, whereas earlier versions had the 4 ceramic rods on either side pointing in opposite directions. Therefore, despite the difference in coefficient of linear thermal expansion for ceramic and stainless steel, heating the decelerator does not lead to severe mechanical stress. Rather, baking results in a movement of the decelerator with respect to the frame, but each of the contact points moves the same distance in the same direction and the crucial alignment of the decelerator is preserved. This simple modification allows us to safely bake the decelerator up to $100{ }^{\circ} \mathrm{C}$. The decelerator has been tested through 10 bake-out cycles with no loss of signal, indicating that alignment is not affected.

\section{Surface preparation and scattering}

After the molecular beam exits the decelerator, it passes through a third $2 \mathrm{~mm}$ aperture into the UHV surface chamber where it is scattered from the prepared surface. The surface chamber is a two tiered vacuum chamber. The upper level houses all surface characterization and preparation equipment including an ion-sputtering gun (STAIB INSTRUMENTS, IG5-C), Auger electron spectrometer (STAIB INSTRUMENTS, ESA-150), gas manifold for deposition of adsorbate gas onto the surface, residual gas analyzer (RGA, SRS RGA-200) for leak detection and thermal desorption measurements, and Kelvin probe (KP Technology, Ltd., UHV-KP010USB) for work function measurements. Surface scattering takes place in the lower level, where MCP detectors (Tectra Physikalische Instrumente $\mathrm{GmbH}, \mathrm{MCP} 050,40 \mathrm{~mm}$, chevron configuration), ion optics, and laser access are mounted.

\section{Target surface}

The sample surface is a $1 \mathrm{~cm}$ diameter cylindrical $\mathrm{Au}$ crystal cut along the (111) plane $(\mathrm{MaTecK})$. The sample is mounted at the end of a 55 in. oxygen free high conductivity (OFHC) copper cold finger connected to a closed-cycle Gifford-McMahon helium cooler (ARS Cryo CS-204AB). The expander and cold finger are mounted on a commercial 4-axis manipulator (VG-Scienta Omniax MXZ800 and MT211B6S) with $800 \mathrm{~mm}$ travel in the vertical axis, $50 \mathrm{~mm}$ travel on the two horizontal axes, and full $360^{\circ}$ rotation around the vertical axis realized with a small differentially pumped rotary feedthrough (DPRF, VG Scienta ZRP100H, DN 100CF), hereafter referred to as the small DPRF, mounted atop the manipulator.

The crystal itself is mounted between two tungsten wires which are connected using stainless steel screws to OFHC copper blocks, see Figure 6. These two OFHC copper blocks are affixed to a central OFHC copper block via $1 \mathrm{~mm}$ thick sapphire disks. The central copper block is then affixed to the end of the cold finger with a single stainless steel 1/4-28 bolt. The sapphire spacers serve two purposes. First, the sapphire is used to electrically insulate the surface from the cold finger, allowing for resistive heating by running current through the tungsten filaments. Second, because the thermal conductivity of sapphire is extraordinarily high at low temperatures and decreases with increasing temperature, the surface is very strongly thermally coupled with the cold finger at low temperatures and only poorly coupled at high temperatures, minimizing the thermal load on the He cooler during sample heating to the highest temperatures needed for annealing. After heating the surface to $1000 \mathrm{~K}$ for $40 \mathrm{~min}$, it takes only $20 \mathrm{~min}$ for the surface to return to $20 \mathrm{~K}$. Crystal temperature is monitored via a chromel-constantan (E-type) thermocouple, the junction of which is placed into a $0.5 \mathrm{~mm}$ diameter hole cut $5 \mathrm{~mm}$ deep in the side of the crystal and staked in with a gold wire. The thermocouple wires run through a double bore $\mathrm{Al}_{2} \mathrm{O}_{3}$ rod mounted within the central copper block to extension wires mounted along the cold finger.

The surface is cleaned by standard sputter-anneal cycles until no contamination is detectable by Auger electron

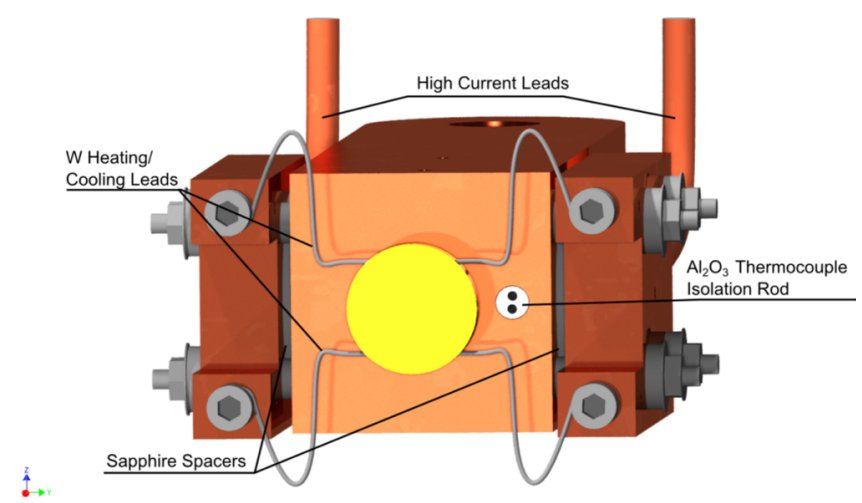

FIG. 6. CAD drawing showing details of the front face of the sample holder. The Au(111) surface is insulated electronically by sapphire spacers. The crystal is suspended between preformed tungsten wires, which also serve as heating and cooling leads. Surface temperatures can be controlled between 19 and $1337 \mathrm{~K}$. 


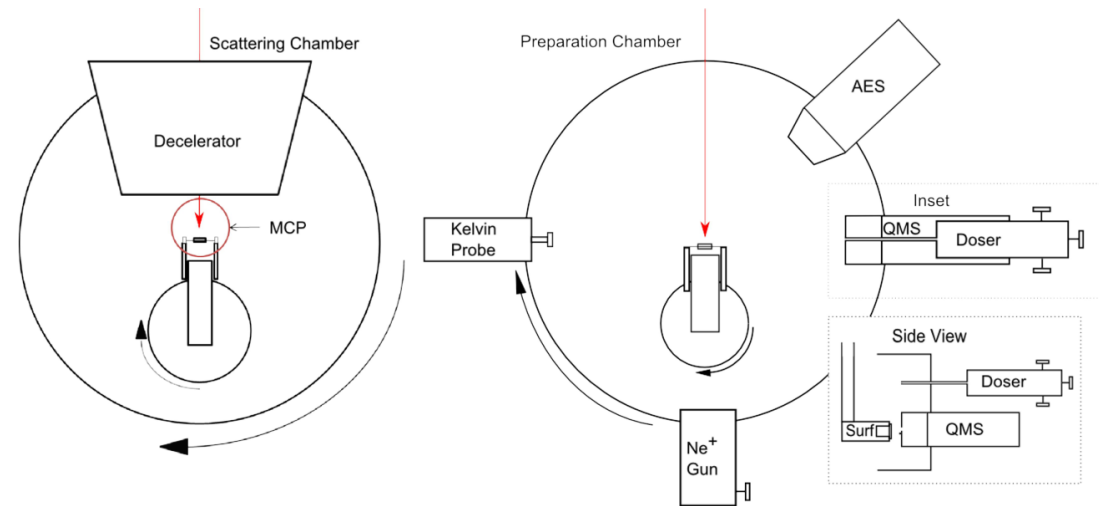

FIG. 7. Left panel shows position of sample surface in front of molecular beam. The red arrow indicates the path of the molecular beam. Rotation of big and small differentially pumped rotary feedthroughs is indicated with black arrows. MCP: microchannel plate detector. Right panel shows preparation chamber. QMS: quadrupole mass spectrometer, doser: leak valve manifold with gas cell for targeted adsorbate dosing, AES: Auger electron spectrometer.

spectroscopy. We clean the surface by sputtering (ca. $25 \mu \mathrm{A} / \mathrm{cm}^{2}$ surface ion current) with $3 \mathrm{kV} \mathrm{Ne}$ ions for 15-20 min and subsequent annealing at $900 \mathrm{~K}$ for $20 \mathrm{~min}$. Our decision to sputter with $\mathrm{Ne}$ rather than Ar ions is due to the low temperature cold finger. After 20 min sputtering with $\mathrm{Ar}$ ions, the chamber regains base pressure only after 40-60 min due to Ar adsorbing and subsequently slowly desorbing from the $20 \mathrm{~K}$ cold finger creating a short term virtual leak. Ne, on the other hand, desorbs quickly from the cold copper and the chamber returns to base pressure within $5 \mathrm{~min}$ after sputtering.

The manipulator is mounted vertically atop a $13.25 \mathrm{in}$. DPRF (Thermionics RNN-1000/MS), hereafter referred to as the big DPRF. The manipulator is mounted off-center from the main vertical axis of rotation in order to allow for a variety of different experimental geometries. As shown in Figure 7, the sample holder is offset from the cold finger so that when the surface is oriented toward the center of the chamber, the front face of the crystal lies on the central rotation axis of the big DPRF. In this way, we can vary scattering angle relative to the molecular beam from $0^{\circ}-90^{\circ}$ by rotating the big DPRF. When characterizing and preparing the surface, we rotate the small DPRF such that the surface points directly away from the center of the chamber and rotate the big DPRF to position the surface in front of the different instruments mounted around the perimeter of the upper tier.

\section{Temperature programmed desorption}

Temperature programmed desorption (TPD) or thermal desorption spectroscopy (TDS) is a common technique in surface science for determining the binding characteristics of adsorbates. The principle is simple: a gas is adsorbed onto a surface at low temperature, the surface is then heated linearly in time and a mass spectrometer is used to measure the partial pressure of desorbed species. When pumping speed is fast enough that approximately no readsorption takes place, the density of detected molecules is directly proportional to the rate of desorption. As the crystal is heated, the rate of desorption increases, resulting in a rise in the density of detected molecules. At some temperature, the population of adsorbed molecules is depleted and the mass spectrometer signal decreases. The shape and position of the peak in the mass spectrometer signal can be used to determine desorption activation energy.

During TPD measurements, we detect desorbed gas using a residual gas analyzer with one modification. Instead of mounting the electron bombardment ionization filament directly in vacuum, as in the off-the-shelf configuration, we have constructed a special nipple which encloses the ionizer in a copper cap, after the design of Feulner and Menzel. ${ }^{20}$ As seen in Figure 8, a $1 \mathrm{~mm}$ diameter thin aperture is centered on the front face of the cylindrical cap. When performing TPD measurements, the front face of the crystal surface is positioned $1.5 \mathrm{~mm}$ from the front of the cap centered on the aperture. This geometrically limits gas entering the ionization area to those particles desorbed from the front face of the crystal. Four larger holes have been drilled around the perimeter of the cap behind the ionizer so that it is pumped into the surface chamber. This setup has two major advantages versus a "naked" ionizer. First, the gas entering the ionization region comes predominantly from the front face of the crystal. Second, the mass spectrometer's sensitivity is enhanced due

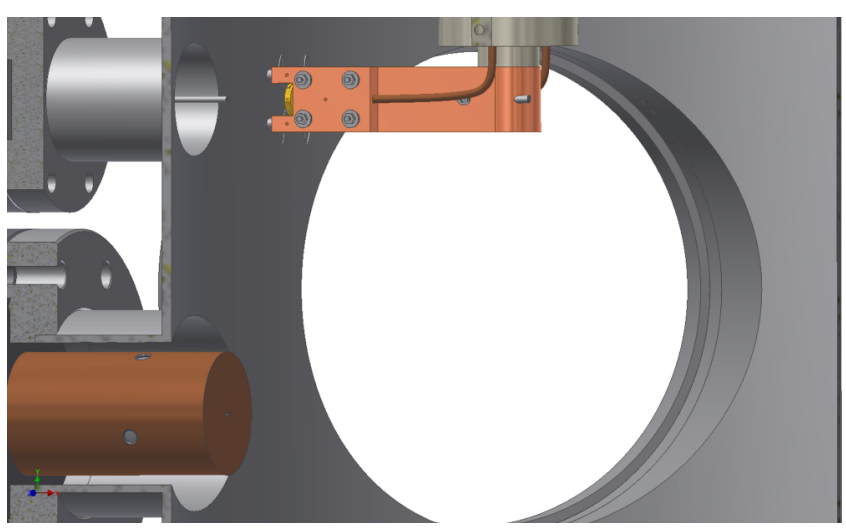

FIG. 8. CAD drawing of surface positioned in front of adsorbate doser. In the lower part of the chamber, the front cap of the RGA is visible. For TPD measurement, the surface is translated downward until the front face of the crystal is ca. $1.5 \mathrm{~mm}$ from the small aperture in the RGA cap. The $1 \mathrm{~mm}$ aperture on the front face of the cylindrical cap limits gas entering the ionization to that originating from the front face of the crystal. The limited pumping speed enhances signal-to-noise ratio by allowing particles to make multiple passes through the ionizer. 
to the fact that the molecules which enter the ionization volume spend an average of $\tau=100 \mathrm{~ms}$ inside the cap before being pumped out into the surface chamber. Increasing $\tau$ allows desorbed molecules to make several passes through the ionizer, thereby significantly increasing signal-to-noise ratio. $^{20}$

We introduce adsorbates onto our surface using a homebuilt surface doser, shown schematically in Figure 7 and as a CAD drawing in Figure 8. Three precision leak valves (MDC Precision Leak, DN40CF/DN16CF) are used to pressurize a gas cell which is connected to the main chamber via an $83 \mathrm{~mm}$ stainless steel tube with an inner diameter of $500 \mu \mathrm{m}$. By pressurizing the gas cell behind the steel tube, we can leak adsorbate gas into the chamber with a narrow, well defined angular distribution. ${ }^{21}$ By positioning the surface $18 \mathrm{~mm}$ from the aperture, $90 \%$ of the flux leaving the tube will first impinge upon the front face of the crystal before scattering into the rest of the chamber. In this way, we can further suppress any spurious peaks that might arise in the TPD spectrum due to adsorbed species desorbing from other parts of the sample holder. By monitoring the pressure in the gas cell and ambient pressure in the chamber during dosing, we are able to determine empirical "recipes" for reproducible dosing with accuracy of \pm 0.1 monolayer (ML).

\section{Kelvin probe work function measurements}

We measure the work function of clean and adsorbate covered surfaces using a scanning Kelvin probe (KP technology, UHVKP020). A Kelvin probe is an appropriate tool for measuring the work function, $\Phi$, of an adsorbate covered surface as it is completely non-destructive and will not desorb even very weakly bound physisorbed particles. The work function measurements follow the procedure developed by Baikie et al $^{22}$ The sample surface is moved very close to a flat $5 \mathrm{~mm}$ diameter reference surface attached to the Kelvin probe, forming a capacitor. The Kelvin probe measures the contact potential difference, $V_{\mathrm{CPD}}$, between the two materials. We then calculate the work function difference using the relation $e V_{\mathrm{CPD}}=-\Delta \Phi$, where $e$ is the charge of an electron and $\Delta \Phi$ is the work function difference between the two surfaces. By calibrating the probe's work function against a known standard, the work function can be determined with $\mathrm{mV}$ accuracy.

\section{Surface scattering and electron detection}

We use resonance enhanced multiphoton ionization (REMPI) spectroscopy to probe the incident beam and detect scattered molecules with quantum-state sensitivity. $\mathrm{CO}^{*}$ molecules can be detected prior to surface collision by $1+1$ REMPI using a frequency doubled pulsed dye laser (Spectra Physics PDL-2, pumped by the second harmonic of a Continuum Powerlite $9010 \mathrm{Nd}$ :YAG laser) resonant at $283 \mathrm{~nm}$ with the $\mathrm{b}^{3} \Sigma^{+} \leftarrow \mathrm{a}^{3} \Pi$ transition. The ions are extracted toward a homebuilt detector consisting of a double MCP stack in a chevron configuration and an extraction grid (91\% transmission, Ni) mounted in the chamber eight $\mathrm{cm}$ below the molecular beam axis. The MCP stack and extraction grid are powered by individual power supplies (Iseg Spezialelektronik GmbH EHQ 105). By controlling the voltage on the extraction grids independently from the detector voltages, we can vary the extraction conditions to optimize collection efficiency while retaining MCP gain conditions. This facilitates much simpler analysis when performing comparative measurements.

A second MCP detector of similar design is mounted four $\mathrm{cm}$ above the molecular beam axis for detecting electrons ejected when $\mathrm{CO}^{*}$ quenches at the surface. ${ }^{13}$ By characterizing the electron emission yield of this process from clean $\mathrm{Au}(111)$, we can monitor the population of our molecular beam pulses. Further, we have a convenient benchmark for comparison with modified surfaces. Shown in the right panel of Figure 9 is a photo of the sample holder, charged particle detectors, and differential pumping wall separating decelerator from surface chamber. The left panel displays a SimION (Scientific Instrument Services, Inc.) trajectory simulation of charged particles originating at various positions relative to surface

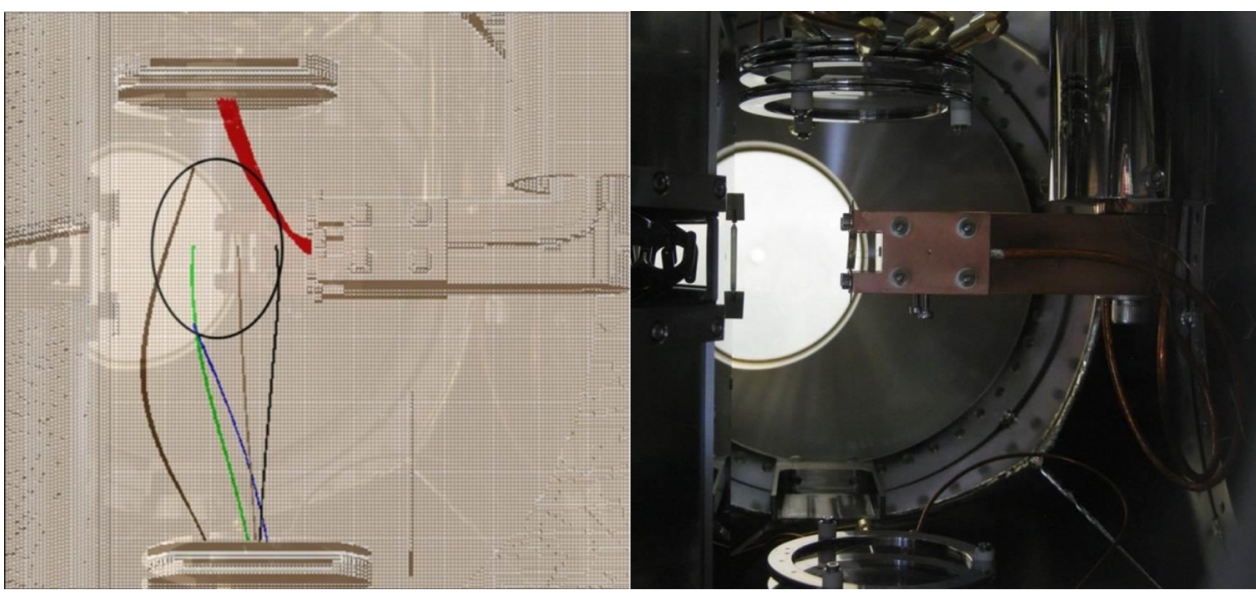

FIG. 9. Left panel: SimION simulation of electrons originating from the surface (red), CO in incident molecular beam pulse at 10 , 20 , and 30 mm from the surface (black, tan, green), and molecules scattered at $35^{\circ}$ relative to front plane of the Au(111) crystal (brown, blue). The black ellipse indicates the large region in which ionized particles can be extracted with $100 \%$ efficiency. Right panel: photograph of surface position in front of molecular beam. MCP detectors are mounted above and below the scattering region. 
and detector. Prior to ionization, the singly charged particles of mass 28 amu travel either toward the surface for those along the molecular beam axis (green, grey, black) or away from the surface for those off the beam axis (brown, blue) with $151.6 \mathrm{~cm}^{-1}(360 \mathrm{~m} / \mathrm{s})$ of translational energy. The ions originating off axis represent molecules scattered from the surface. $100 \%$ extraction is possible over a large detection volume, indicated in Figure 9 with the black ellipse, allowing for efficient time-of-flight and angular distribution measurements. Scanning the position of the focus of the ionization laser can be accomplished by means of a periscope outside the chamber. A $100 \mathrm{~mm}$ UV fused silica window is mounted on each side of the surface scattering region for laser access.

As can be seen in the photo in Figure 9, laser access to the last few stages of the decelerator has been achieved by mounting a special end cap on the surface side of the decelerator chamber which extends into the surface chamber. Two UV fused silica windows are installed at Brewster's angle on either side of the cap to allow laser access to the molecular beam for six electrode stages before the end of the decelerator. This allows us to perform optical excitation and de-excitation experiments with molecules before the $\mathrm{CO}^{*}$ pulse has left the decelerator. Then, according to simulations, by over focusing with the last decelerator stages we can deflect the remaining metastables away from the beam axis. In this way, we can prepare a beam of purified electronic ground state molecules for scattering from the surface. Alternatively, we can use the metastable quenching electron signal for shot-to-shot normalization of beam intensity.

\section{SELECTED RESULTS}

This section presents experimental results that demonstrate the capabilities and performance characteristics of the new machine. Our apparatus is designed to examine energy exchange between quantum-state selected molecules and well characterized surfaces at extremely low incidence energies.

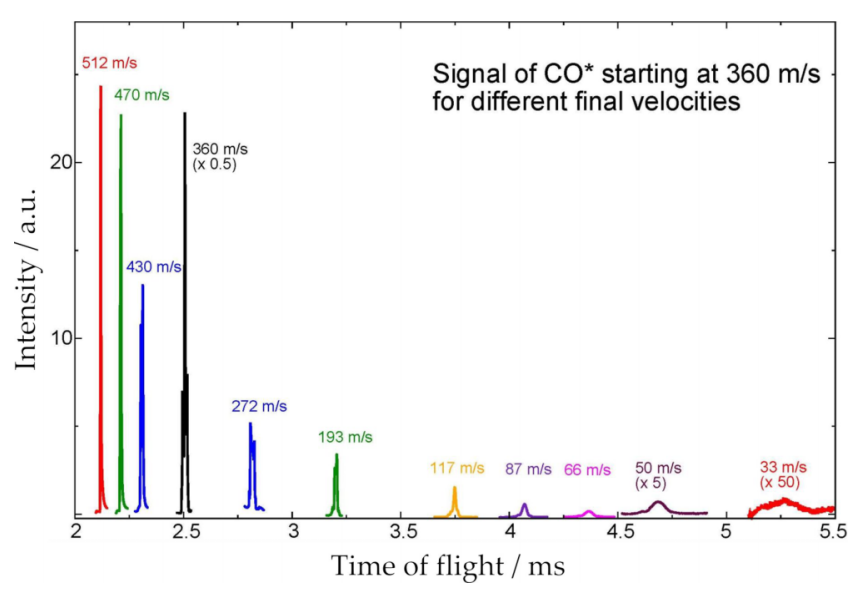

FIG. 10. Time resolved signal of ejected electrons as $\mathrm{CO}^{*}$ impinges on a $\mathrm{Au}(111)$ surface under different conditions of velocity manipulation. Electron signal is detected on a MCP detector. The traces show the time-of-flight distribution of the neutral molecules.
TABLE I. Calculated final velocities, $v_{\mathrm{f}}$, longitudinal velocity spread, $\Delta v_{\mathrm{f}}$, collision kinetic energy, $E_{\text {coll }}$, and collision energy spread, $\Delta E_{\text {coll }}$ for initial beam velocities of $360 \mathrm{~m} / \mathrm{s}^{23}$

\begin{tabular}{ccccc}
\hline \hline$\varphi_{0}(\mathrm{deg})$ & $v_{\mathrm{f}}(\mathrm{m} / \mathrm{s})$ & $\Delta v_{\mathrm{f}}(\mathrm{m} / \mathrm{s})$ & $E_{\mathrm{coll}}\left(\mathrm{cm}^{-1}\right)$ & $\Delta E_{\mathrm{coll}}\left(\mathrm{cm}^{-1}\right)$ \\
\hline-90 & 512 & 4 & 307 & 5 \\
-50 & 470 & 11 & 259 & 12 \\
-30 & 430 & 16 & 216 & 16 \\
0 & 360 & 14 & 152 & 12 \\
30 & 272 & 16 & 87 & 10 \\
40 & 235 & 14 & 65 & 7 \\
50 & 193 & 11 & 44 & 5 \\
60 & 145 & 8 & 25 & 3 \\
70 & 87 & 6 & 9 & 1 \\
75 & 50 & 4 & 3 & 1 \\
\hline \hline
\end{tabular}

\section{A. Deceleration and acceleration}

Figure 10 shows electrons ejected from an atomically clean $\mathrm{Au}(111)$ surface during the quenching of an impinging beam of $\mathrm{CO}^{*}$ molecules at velocities from 33 to $512 \mathrm{~m} / \mathrm{s}$. This corresponds to $1.3-306.7 \mathrm{~cm}^{-1}$ collision energy when the molecule strikes the surface. The signal is recorded on a MCP detector as described in Sec. II C 4. The corresponding velocities and kinetic collision energies, $v_{\mathrm{f}}$ and $E_{\text {coll }}$, and associated half-widths, $\Delta v_{\mathrm{f}}$ and $\Delta E_{\text {coll }}$, have been calculated using three dimensional trajectory simulations and are reported in Table I. ${ }^{23}$ Access to such low incident translational energies with extremely narrow velocity distributions enables experiments which probe energy transfer at the gas-surface interface with unprecedented resolution.

\section{B. Work function measurements}

Shown in Figure 11 are measurements of the adsorbate induced change in the surface work function, $\Delta \Phi$, of $\mathrm{Au}(111)$ with various coverages of noble gas adsorbate atoms. Samples were prepared by exposing a $19 \mathrm{~K} \mathrm{Au}(111)$ surface with a

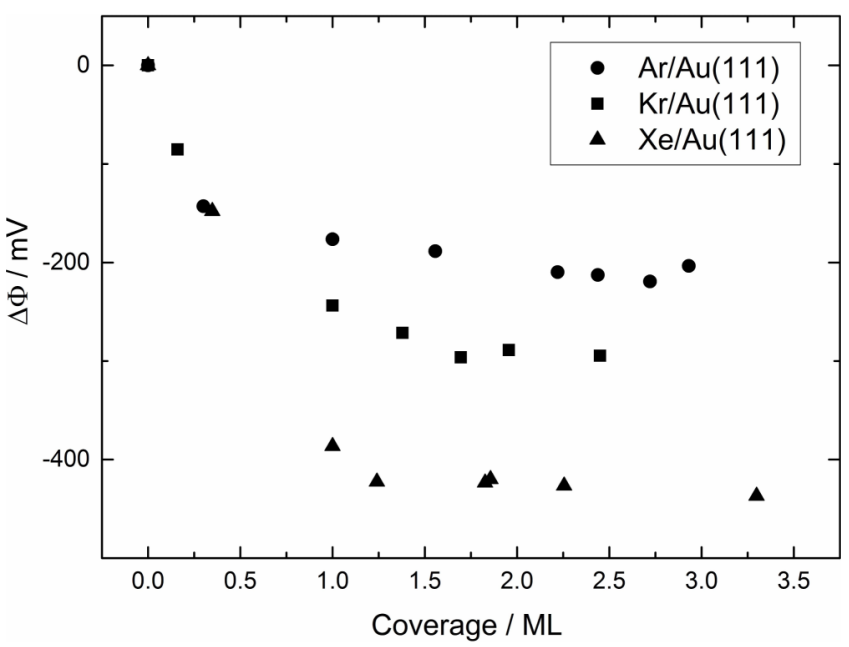

FIG. 11. Change in the work function, $\Phi$, of a $\mathrm{Au}(111)$ surface at various adsorbate coverages. One ML results in a work function change of $-180 \mathrm{meV}$, $-250 \mathrm{meV}$, and $-380 \mathrm{meV}$ for argon (circles), krypton (squares), and xenon (triangles). Further adsorption of each gas results in a much smaller change in $\Phi$. 
controlled dose of adsorbate. The work function of the rare gas/Au(111) surface was then measured using the Kelvin probe. Exact coverage was subsequently determined by performing a TPD experiment on the same surface.

It is shown in Figure 11 that an adsorbate monolayer results in a significant change in $\Phi$ for all measured gases. A change of $-180 \mathrm{meV},-250 \mathrm{meV}$, and $-380 \mathrm{meV}$ is measured for adsorbed argon, krypton, and xenon monolayers, respectively. Further adsorption results in relatively small work function changes for each gas. Since $\Phi$ is a measure of how much energy is necessary to remove an electron from a solid into vacuum, it is a critical parameter in many energy transfer processes. The ability to prepare and characterize such complex surfaces enables study of many different molecule/surface systems with complete control.

\section{Temperature programmed desorption}

Temperature programmed desorption experiments are used to determine the binding energy of different adsorbate/surface systems and are a convenient method of determining exact adsorbate coverages. Shown in Figure 12 are TPD spectra of argon, krypton, and xenon desorbing from $\mathrm{Au}(111)$. Initial coverage of at least $3 \mathrm{ML}$ for all gases is enough to populate all three desorption states. In each spectrum, the peak designated $\gamma$ is attributed to the monolayer of atoms in direct contact with the surface, $\beta$ to the second layer or bilayer, and the non-saturable $\alpha$ peak to the third and all further overlayers.

In each case, a $19 \mathrm{~K} \mathrm{Au}(111)$ surface was exposed to rare gas leaked into the surface chamber through the narrow stainless steel nozzle as shown in the CAD drawing of Figure 8. Due to the narrow angular distribution of the

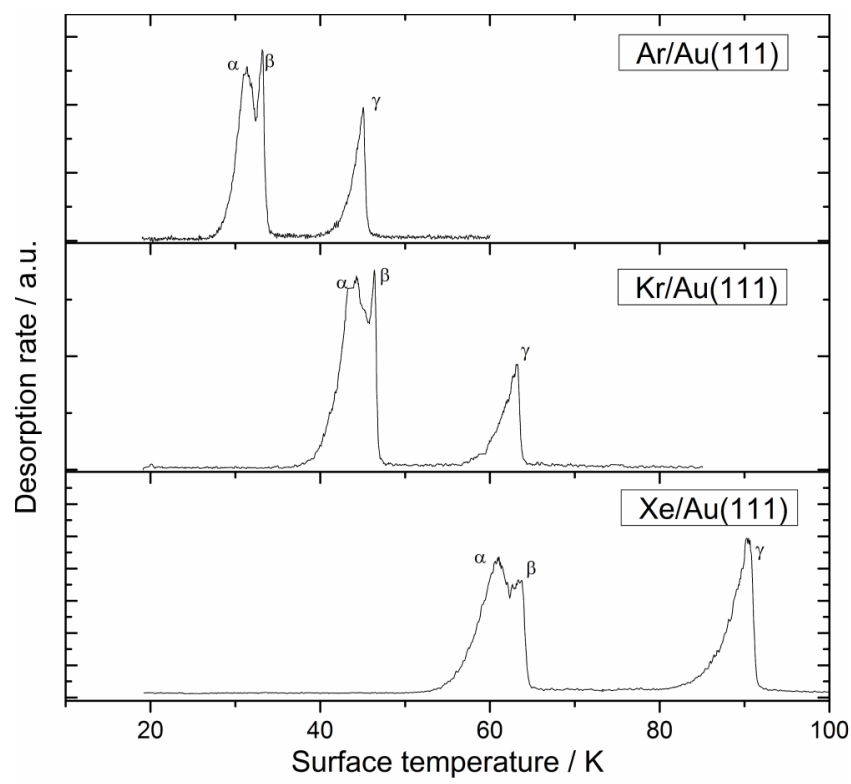

FIG. 12. Temperature programmed desorption spectra of $\mathrm{Ar}, \mathrm{Kr}$, and $\mathrm{Xe}$ from $\mathrm{Au}(111)$ with initial coverage of $3.6 \mathrm{ML}$, 4.5 ML, and 3.5 ML, respectively. Experimental conditions were chosen such that re-adsorption probability was negligible for all scans. All scans were recorded at a heating rate of $0.5 \mathrm{~K} / \mathrm{s}$. For each scan, $\alpha$ corresponds to the non-saturable over layer peak, $\beta$ to the bilayer peak, and $\gamma$ to the monolayer peak.
TABLE II. Desorption activation energies $E_{\mathrm{des}}$ and desorption order for argon, krypton, and xenon on $\mathrm{Au}(111)$.

\begin{tabular}{cc}
\hline \hline Adsorbate & $E_{\text {des }}(\mathrm{eV})$ \\
\hline $\mathrm{Ar}$ & $0.14 \pm 0.02$ \\
$\mathrm{Kr}$ & $0.19 \pm 0.02$ \\
$\mathrm{Xe}$ & $0.24 \pm 0.02$ \\
\hline \hline
\end{tabular}

particles emitted from the nozzle, the front face of the crystal can be exposed to arbitrarily high adsorbate doses within a convenient timescale while maintaining ambient chamber pressure of $<2 \times 10^{-9}$ Torr. This is necessary when dosing with aggressive species such as $\mathrm{HCl}$ to preserve the delicate instruments in the UHV chamber.

The monolayer peak shape indicates a zero-order desorption kinetics for all rare gases. This is in good accordance with previous measurements on rare gas metal systems. ${ }^{24}$ We analyze the monolayer desorption kinetics based on the Polanyi-Wigner equation

$$
r=-\frac{d \theta}{d t}=v(\theta) \theta^{n} \exp \left(-E_{\mathrm{des}}(\theta) / R T\right) .
$$

Here, $\theta$ is the coverage, $t$ the time, $v$ the pre-exponential factor, $E_{\mathrm{des}}$ the desorption activation energy, $R$ the molar gas constant, $T$ the temperature, and $n$ the desorption order. Following the approach presented by Niemantsverdriet et al., we vary $n$ until an Arrhenius plot of $\ln \left(r / \theta^{n}\right)$ vs. $1 / T$ of the monolayer leading edge is a straight line. ${ }^{25}$ The Arrhenius plot yields the desorption activation energy $E_{\text {des }}$. The $E_{\text {des }}$ values for $\mathrm{Ar}, \mathrm{Kr}$, and $\mathrm{Xe}$ on $\mathrm{Au}(111)$ are displayed in Table II. Due to the increasing polarizability of the noble gases going down the periodic table, $\mathrm{Xe}$ atoms are more strongly physisorbed to the $\mathrm{Au}(111)$ surface than are $\mathrm{Kr}$ and $\mathrm{Ar}$ atoms.

\section{SUMMARY AND PROSPECTUS}

The interplay of different fields on the boundaries between chemistry and physics has helped to develop a sophisticated understanding of surface dynamics. ${ }^{9}{ }^{10,26,27}$ However, due to limited experimental data, no comprehensive picture has yet been drawn. Among others, the role of electronically nonadiabatic coupling between surface and reactants, the role of the phonon bath on surface dynamics, the role of electronically excited molecules, and the role of different interaction mechanisms (trapping/desorption vs. direct mechanisms) are still debated in the scientific community. ${ }^{9,28,29}$ Combining the molecular beam-surface scattering technique with the field of decelerated molecules offers the possibility to perform remarkable new experiments. ${ }^{4,29,30}$ Complete control over translational, electronic, rotational, and vibrational energy of the incoming molecules will enable studies of sticking processes ${ }^{31}$ vibrationally promoted surface reactions, ${ }^{32}$ vibrational energy transfer, ${ }^{6}$ and surface electron excitation ${ }^{7}$ with access to energetic regimes that have thus far been experimentally unfeasible.

For this purpose, we have designed and constructed a Stark decelerator-based surface scattering apparatus. The performance of the molecular decelerator has been demon- 
strated by monitoring electron emission from the quenching of $\mathrm{CO}^{*}$ molecules at a $\mathrm{Au}(111)$ surface over a wide range of incident translational energies. The capabilities of the sample preparation equipment have been demonstrated by TPD and work function studies of rare gas adsorbates on the $\mathrm{Au}(111)$ surface. The flexibility shown by the presented results demonstrates the utility and potential of this new apparatus. Studies in the near future will investigate the interaction between complex surfaces and ground state $\mathrm{CO}$. In the long term, the apparatus can be used to scatter a variety of molecules which are compatible with Stark deceleration. ${ }^{16}$ By obtaining complete control over the molecular and surface degrees of freedom, we hope to provide benchmark measurements for a broad range of systems, allowing for close comparison with state-of-the-art theory.

\section{ACKNOWLEDGMENTS}

We would like to thank the National Science Foundation, the Deutsche Forschungsgemeinschaft, and the Alexander von Humboldt Foundation for financial support. R.J.V.W. gratefully acknowledges a Ph.D. fellowship granted by the Fonds der Chemischen Industrie.

${ }^{1}$ C. R. Arumainayagam and R. J. Madix, Prog. Surf. Sci. 38, 1 (1991).

${ }^{2}$ A. M. Wodtke, D. Matsiev, and D. J. Auerbach, Prog. Surf. Sci. 83, 167 (2008).

${ }^{3}$ C. T. Rettner, D. J. Auerbach, J. C. Tully, and A. W. Kleyn, J. Phys. Chem. 100, 13021 (1996).

${ }^{4}$ M. Silva, R. Jongma, R. W. Field, and A. M. Wodtke, Annu. Rev. Phys. Chem. 52, 811 (2001).

${ }^{5}$ K. Golibrzuch, A. Kandratsenka, I. Rahinov, R. Cooper, D. J. Auerbach, A. M. Wodtke, and C. Bartels, J. Phys. Chem. A 117, 7091 (2013).

${ }^{6}$ Y. Huang, C. T. Rettner, D. J. Auerbach, and A. M. Wodtke, Science 290 , 111 (2000).

${ }^{7}$ N. H. Nahler, J. D. White, J. Larue, D. J. Auerbach, and A. M. Wodtke, Science 321, 1191 (2008).
${ }^{8}$ T. Schaefer, N. Bartels, K. Golibrzuch, C. Bartels, H. Kockert, D. J. Auerbach, T. N. Kitsopoulos, and A. M. Wodtke, Phys. Chem. Chem. Phys. 15, 1863 (2013).

${ }^{9}$ N. Shenvi, S. Roy, and J. C. Tully, Science 326, 829 (2009).

${ }^{10}$ N. Shenvi, S. Roy, and J. C. Tully, J. Chem. Phys. 130, 174107 (2009).

${ }^{11}$ J. D. White, J. Chen, D. Matsiev, D. J. Auerbach, and A. M. Wodtke, J. Chem. Phys. 124, 064702 (2006).

${ }^{12}$ B. D. Kay, T. D. Raymond, and M. E. Coltrin, Phys. Rev. Lett. 59, 2792 (1987).

${ }^{13}$ F. Graetz, D. P. Engelhart, R. J. V. Wagner, H. Haak, G. Meijer, A. M. Wodtke, and T. Schaefer, Phys. Chem. Chem. Phys. 15, 14951 (2013).

${ }^{14}$ F. Graetz, D. P. Engelhart, R. J. V. Wagner, G. Meijer, A. M. Wodtke, and T. Schaefer, J. Chem. Phys. 141, 044712 (2014).

${ }^{15}$ L. Velarde, D. P. Engelhart, D. Matsiev, J. LaRue, D. J. Auerbach, and A. M. Wodtke, Rev. Sci. Instrum. 81, 063106 (2010)

${ }^{16}$ H. L. Bethlem, F. M. H. Crompvoets, R. T. Jongma, S. Y. T. van de Meerakker, and G. Meijer, Phys. Rev. A 65, 053416 (2002).

${ }^{17}$ C. E. Heiner, H. L. Bethlem, and G. Meijer, Phys. Chem. Chem. Phys. 8, 2666 (2006)

${ }^{18}$ S. Y. T. van de Meerakker, H. L. Bethlem, and G. Meijer, Nat. Phys. 4, 595 (2008).

${ }^{19}$ S. Y. T. van de Meerakker, N. Vanhaecke, H. L. Bethlem, and G. Meijer, Phys. Rev. A 73, 023401 (2006).

${ }^{20}$ P. Feulner and D. Menzel, J. Vac. Sci. Technol. 17, 662 (1980).

${ }^{21} \mathrm{G}$. Scoles, Atomic and Molecular Beam Methods (Oxford University Press, 1988).

${ }^{22}$ I. D. Baikie, K. O. Vanderwerf, H. Oerbekke, J. Broeze, and A. Vansilfhout, Rev. Sci. Instrum. 60, 930 (1989).

${ }^{23}$ F. Grätz, Ph.D. dissertation, Freie Universität Berlin, 2014.

${ }^{24}$ H. Schlichting and D. Menzel, Surf. Sci. 272, 27 (1992).

${ }^{25}$ J. W. Niemantsverdriet, P. Dolle, K. Markert, and K. Wandelt, J. Vac. Sci. Technol., A 5, 875 (1987).

${ }^{26}$ G. J. Kroes, A. Gross, E. J. Baerends, M. Scheffler, and D. A. McCormack, Acc. Chem. Res. 35, 193 (2002).

${ }^{27}$ D. Troya and G. C. Schatz, Int. Rev. Phys. Chem. 23, 341 (2004).

${ }^{28}$ D. J. Auerbach, Science 294, 2488 (2001).

${ }^{29}$ A. M. Wodtke, J. C. Tully, and D. J. Auerbach, Int. Rev. Phys. Chem. 23, 513 (2004).

${ }^{30}$ C. Kittrell, E. Abramson, J. L. Kinsey, S. A. McDonald, D. E. Reisner, R. W. Field, and D. H. Katayama, J. Chem. Phys. 75, 2056 (1981).

${ }^{31}$ C. T. Rettner, D. J. Auerbach, and H. A. Michelsen, Phys. Rev. Lett. 68, 1164 (1992).

${ }^{32}$ H. Hou, Y. Huang, S. J. Gulding, C. T. Rettner, D. J. Auerbach, and A. M. Wodtke, Science 284, 1647 (1999). 